\title{
Application of Wavelet Analysis and Neural Network in Fault Diagnosis of Rolling Bearing
}

\author{
Li Xinli" a, Yao Wanye ${ }^{2, b}$ and Yang $X^{1}{ }^{3, c}{ }^{3, c}$ \\ and Zhou Qingjie ${ }^{4, d}$ \\ ${ }^{1}$ School Of Control And Computer Engineering, North China Electric Power \\ University,Baoding,Hebei,071003,China \\ ${ }^{4}$ Beijing Join Bright digital power technology Co,Ltd, Beijing ,100085,China \\ a601279228@qq.com, byaowanye@163.com, cwangjianming010@126.com
}

Keywords: rolling bearings; fault diagnosis; wavelet packet analysis; neural network

\begin{abstract}
In this paper, a fault-diagnosis method is proposed for generator rolling bearings based on wavelet packet analysis and neural network. Acquisition of wind farm rolling bearings real-time signal under different conditions.Firstly, decomposes vibration acceleration signals use wavelet packets analysis, make the original vibration signal decomposed into different frequency bands, then calculate the energy values, so extracts energy values of various vibration signal to construct fault eigenvector; which use as the input of the neural network. Then, by the parameter setting created a BP neural network ; in order to make the network has memory classification function we need training the network.Finally, the test sample put into the already trained BP get the fault pattern recognition. Using the wind farm real-time data for simulation experimental, the results show that the fault diagnosis model of high precision, can make a fast and effective fault diagnosis for rolling bearings.
\end{abstract}

\section{Introduction}

Rolling generator is one of the most widely used parts of rotating machinery, its operating status can directly affect the work performance of the machine; once a fault occurs, it will result a huge losses, so how to quickly find the failure and in timely to maintenance of fault diagnosis system is the urgent demand of the current industrial development. When the rolling bearing failure, the vibration acceleration signal will changed, so can using the wavelet packet decomposition get energy distribution in different frequency reflected bearing operating status information, which is one of the more common method ${ }^{[1]}$.However, the traditional wavelet analysis can not clearly distinguish the frequency of the high portion, but when the bearing failure the high frequency band decomposition of vibration signal is very important, therefore it is proposed for low and high frequency portion of the signal can be find decomposition wavelet packet fault feature extraction method ${ }^{[2]}$. After extract fault feature we need using intelligent fault diagnosis method. In recent years, with the rapid development of artificial intelligence technology a fault diagnosis method based on knowledge is widely used, because this method don trequire precise mathematical model for the object, but has some smart characteristics ${ }^{[3]}$. Knowledge based fault diagnosis method can be divided into: expert system fault diagnosis: fuzzy fault diagnosis; fault tree for fault diagnosis; neural network fault diagnosis ${ }^{[4]}$. Neural network fault diagnosis method due to have parallel computing, self-organization and nonlinear mapping ability, so in fault diagnosis area has been widely applied $^{[5]}$. Compared with other fault diagnosis methods the neural network has better versatility, effectiveness and generalization ability, it has been widely used in the fault diagnosis classification.

This paper collection the rolling bearings three states of vibration signal: normal operation, bearing inner ring fault and outer race fault. Using wavelet packet method get the fault features. Then use BP neural network fault diagnosis method for identifying the fault category, which is the cause of the failure. 


\section{Wavelet packet analysis and algorithms}

Wavelet packet analysis can simultaneously divided signal into multiple levels of low and high frequency to achieve accurate signal analysis; it can decompose the original signal with any time-frequency resolution, each band signal energy spectrum contains detailed information of the original signal, which can extract the signal characteristic vector in different frequency bands ${ }^{[6]}$. Wavelet Packet Algorithm ${ }^{[7]}$ :

(1)The vibration acceleration signal wavelet packet decomposition

The vibration signal of bearings for 3 layer wavelet decomposition, extraction layer 3 from low frequency to high frequency 8 sub-bands of wavelet packet coefficients $\left(x_{3}^{0}, x_{3}^{1}, x_{3}^{2}, x_{3}^{3}, x_{3}^{4}, x_{3}^{5}, x_{3}^{6}, x_{3}^{7}\right)$ 。

(2)Wavelet packet decomposition coefficients reconstruction

Reconstructed signal representation $S_{3 i}(i=0,1 \ldots 7)$, total signal $\mathrm{S}$ is :

$S=S_{30}+S_{31}+S_{32}+S_{33}+S_{34}+S_{35}+S_{36}+S_{37}$

(3)Find the total energy of each band signal

The reconstructed signal $S_{3 i}$ correspond to the energy values is $E_{3 i}(i=0,1 \ldots 7)$,

$E_{3 i}=\int\left|S_{3 i}\right|^{2} d t=\sum_{k=1}^{n}\left|x_{i k}\right|^{2}, x_{i k}(i=0,1 . .7, k=1,2 \ldots n)$ represents the reconstructed signal amplitude of the discrete points.

(4)Structural features vector

Larger changes within each band signal energy when a failure occurs, according to this

characteristic structure in the vector: $R=\left[E_{30}, E_{31}, E_{32}, E_{33}, E_{34}, E_{35}, E_{36}, E_{37}\right]$

(5)Energy normalized of feature vectors

The total energy of the signal is $E=\sum_{i=0}^{2^{i}-1} E_{i k}$, after the energy-normalized obtained the vibration signal eigenvector $R^{*}: R^{*}=\left[E_{30}, E_{31}, E_{32}, E_{33}, E_{34}, E_{35}, E_{36}, E_{37}\right] / E$.

\section{BP neural network}

BP neural network is a multilayer feedforward neural network, the features of the network is signals prior to transmission, the error back to pass ${ }^{[8]}$.

BP neural network training process as follows ${ }^{[9]}$ :

(1)Network initialization. According to the number of input and output samples determine the input layer nodes $\mathrm{m}$, hidden layer nodes $\mathrm{h}$ and the output layer nodes $\mathrm{n}$; the weights $\omega_{i j}$ and $\omega_{j k}$, the layer threshold is a, b.

(2)Calculate the hidden layer output $\mathrm{H}: H=f\left(\sum_{i=1}^{n} \omega_{i j} x_{i}-a_{j}\right), j=1,2 \ldots h$.

(3)Calculate the output layer predict output O : $O_{k}=\sum_{j=1}^{h} H_{j} \omega_{j k}-b_{k}, k=1,2 \ldots n$.

(4)Error calculation: $e_{k}=Y_{k}-O_{k}, k=1,2 \ldots n . \mathrm{O}$ is predicted output, $\mathrm{Y}$ is actual output.

(5)According to error $e$ adjust the network connection weights and threshold:

$$
\begin{aligned}
& \omega_{i j}=\omega_{i j}+\eta H_{j}\left(1-H_{j}\right) x(i) \sum_{k=1}^{m} \omega_{j k} e_{k}, \omega_{j k}=\omega_{j k}+\eta H_{j} e_{k} . \\
& a_{j}=a_{j}+\eta H_{j}\left(1-H_{j}\right) \sum_{k=1}^{m} \omega_{j k} e_{k}, j=1,2 \ldots m \quad, b_{k}=b_{k}+e_{k}, \quad k=1,2 \ldots n .
\end{aligned}
$$


(6)Determine whether the algorithm is finished, if not the end, return to step 2

\section{Application of wavelet analysis and neural network in fault diagnosis of rolling bearing}

The basic idea of wavelet analysis and BP neural network in fault diagnosis of rolling bearing is: firstly using wavelet packet algorithm for vibration acceleration signal of rolling bearing 3 layer decomposition, make the vibration signal is decomposed into separate bands, and then reconstructed the signal of each band, therefore the signal energy variation within different frequency bands reflects rolling bearing different operating states.so we can extracted wavelet packet energy of each band as a feature vector; the extracted feature vectors are used as input of the BP neural network, the type of fault as a neural network output. Configured the BP neural network Parameter, then training the network make it has association and memory function; after the training is completed, the test sample into the already trained BP network for fault type identification. Fault diagnosis process shown in fig. 1 :

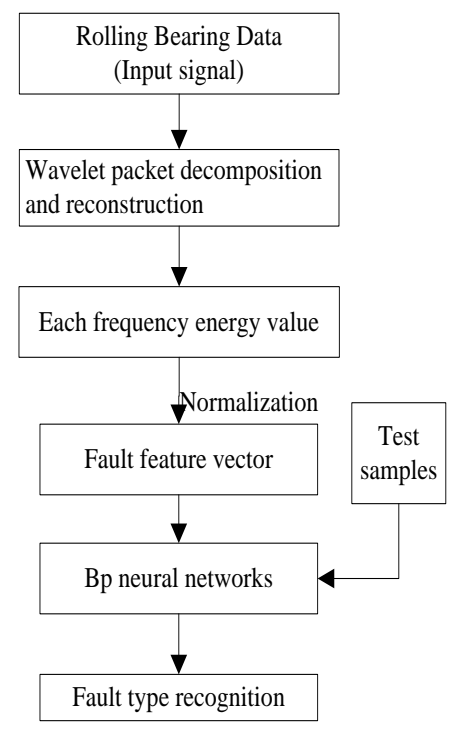

Fig1 Fault diagnosis process

\subsection{Fault feature extraction}

Select 200 rolling bearing vibration data from a historical database of a wind power plant, the sampling frequency is $2000 \mathrm{HZ}$. Data includes rolling bearing normal operating, bearing outer race fault, inner race fault data is50,respectivly. Which of 150 training samples, 50 testing samples.

Bearing normal operating, inner race fault and outer race fault time domain diagram shown in Fig. 2. For 3 Layer wavelet packet of bearing vibration signal:

$\mathrm{x}=\operatorname{wpdec}(\mathrm{s}, 3, \mathrm{db} 10$ '), Where " $\mathrm{s}$ " represents a decomposition signal ,db10 is wavelet packet function; From the results of the decomposition select 3 layer, get the 8 sub-frequency component of Characteristic values from low to high frequency.

Fig. 3 is the wavelet packet frequency of bearing normal operation.Fig. 4 is the wavelet packet frequency of bearing inner ring fault.Fig. 5 is the wavelet packet frequency of bearing outer ring fault. 

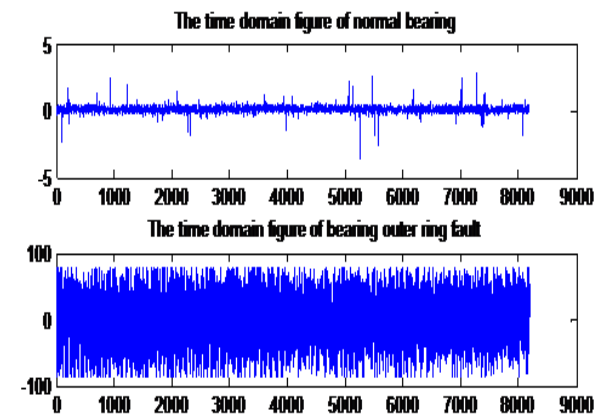

The time domain fygue of beaing inne ring fall

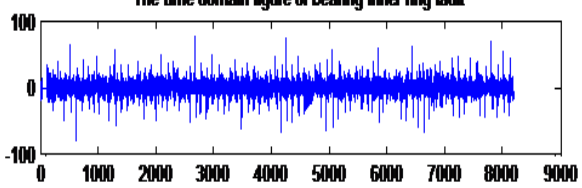

Fig.2 Time-domain diagram

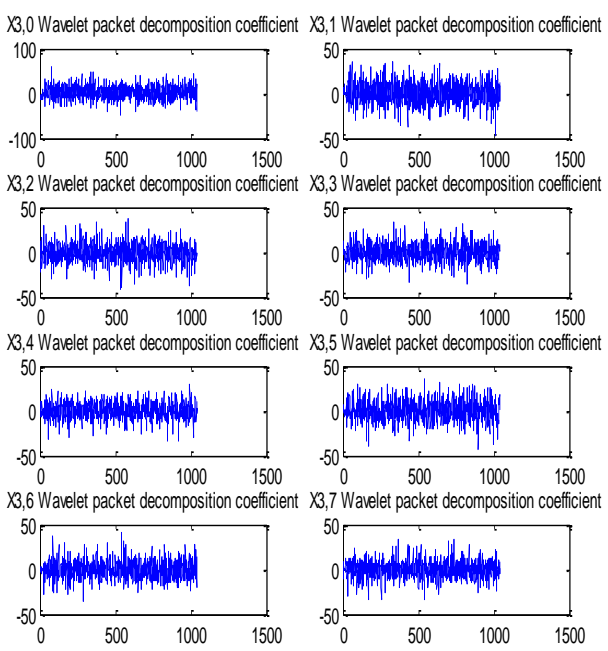

Fig.4wavelet packet frequency of bearing inner ring fault

Form the fig.3,fig. 4 and fig. 5 we can see that: the frequency bands has different signal energy value reflects the characteristics of the operating state, the signal reconstructed as $S_{i}$, then calculate the total energy of each band signal $E_{3 i}, \mathrm{E}(\mathrm{i})=\operatorname{norm}(\operatorname{wpcoef}(\mathrm{t},[\mathrm{n}, \mathrm{i}-1]), 2) * \operatorname{norm}(\operatorname{wpcoef}(\mathrm{t},[\mathrm{n}, \mathrm{i}-1]), 2)$; Some feature vector is shown in table1, which normal operation is represented by 0 ; inner ring fault is represented by 1 ; outer ring fault is represented by 2 ;

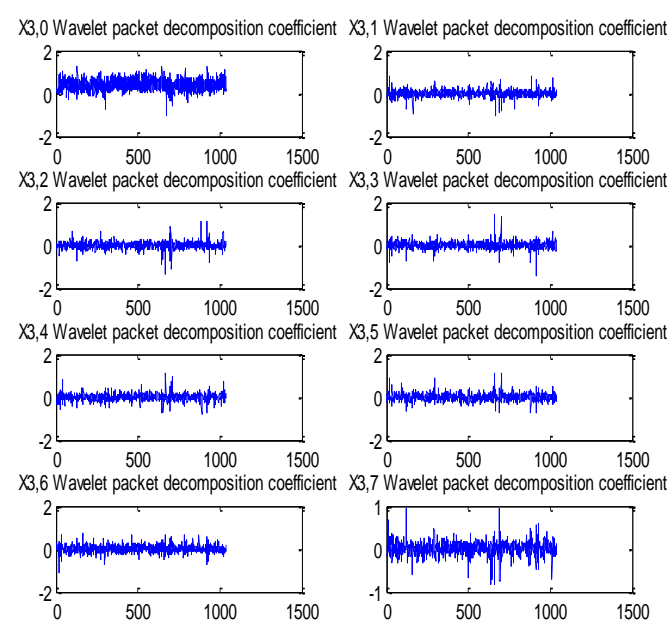

Fig. 3 wavelet packet frequency of bearing normal operation

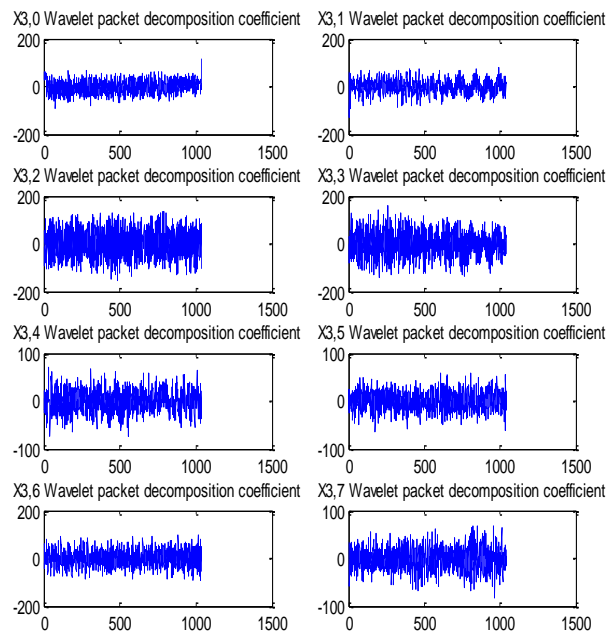

Fig.5wavelet packet frequency of bearing outer ring fault 
Table 1 Feature vector

\begin{tabular}{|c|c|c|c|c|c|c|c|c|c|}
\hline \multirow{2}{*}{$\begin{array}{l}\text { Training } \\
\text { sample }\end{array}$} & \multicolumn{8}{|c|}{ Feature vector } & \multirow{2}{*}{$\begin{array}{l}\text { Fault } \\
\text { type }\end{array}$} \\
\hline & $R_{0}^{*}$ & $R_{0}^{*}$ & $R_{0}^{*}$ & $R_{0}^{*}$ & $R_{0}^{*}$ & $R_{0}^{*}$ & $R_{0}^{*}$ & $R_{0}^{*}$ & \\
\hline 1 & 0.7510 & 0.5001 & 0.4930 & 0.5334 & 0.5555 & 0.4836 & 0.4961 & 0.6040 & 0 \\
\hline 2 & 0.2031 & 0.0374 & 0.0354 & 0.0390 & 0.0406 & 0.0391 & 0.0336 & 0.0380 & 0 \\
\hline 3 & 0.1708 & 0.0283 & 0.0260 & 0.0253 & 0.0271 & 0.0287 & 0.0232 & 0.0347 & 0 \\
\hline 4 & 0.4607 & 0.1952 & 0.2143 & 0.2403 & 0.2016 & 0.2175 & 0.1859 & 0.2399 & 0 \\
\hline 5 & 0.7014 & 0.8011 & 0.6280 & 0.7902 & 0.9910 & 0.8002 & 0.2114 & 0.0012 & 1 \\
\hline 6 & 0.839 & 0.7904 & 0.4197 & 0.4776 & 0.0802 & 0.9978 & 0.3009 & 0.0011 & 1 \\
\hline 7 & 0.9918 & 0.7950 & 0.8085 & 0.3459 & 0.0042 & 0.2703 & 0.2108 & 0.0009 & 1 \\
\hline 7 & 0.0087 & 0.2998 & 0.3801 & 0.9911 & 0.5002 & 0.1703 & 0.1804 & 0.0010 & 2 \\
\hline 9 & 0.9214 & 0.3714 & 0.0308 & 0.1204 & 0.0022 & 0.0078 & 0.1908 & 0.0608 & 2 \\
\hline 10 & 0.9910 & 0.3104 & 0.0017 & 0.2001 & 0.0028 & 0.1709 & 0.1789 & 0.1901 & 2 \\
\hline
\end{tabular}

\subsection{Build fault diagnosis model}

(1)Set network parameters

According the wavelet packet get attribute simples is 8 , so the input layer of the neural network innum $=8$, the experiment is the bearing three different states, so neural network output layer outnum $=3$, the hidden layer node midnum $=12$. after parameter setting, initialize the network weights $\omega_{1}, \omega_{2}$ and thresholds $b_{1}, b_{2}$;

(2)Adjustment weights and threshold

Reverse transfer function of neural networks is continuously adjusted the process of error weights, the program to achieve is following:

$\mathrm{dw} 1(\mathrm{k}, \mathrm{j})=\mathrm{FI}(\mathrm{j}) * \mathrm{x}(\mathrm{k}) *(\mathrm{e}(1) * \mathrm{w} 2(\mathrm{j}, 1)+\mathrm{e}(2) * \mathrm{w} 2(\mathrm{j}, 2)+\mathrm{e}(3) * \mathrm{w} 2(\mathrm{j}, 3))$;

$d b 1(j)=F I(j) *(e(1) * w 2(j, 1)+e(2) * w 2(j, 2)+e(3) * w 2(j, 3))$;

(3)The actual fault type and the wavelet packet-BP neural network prediction fault type, the comparative results shown in fig.6.the classification error shown in fig.7:

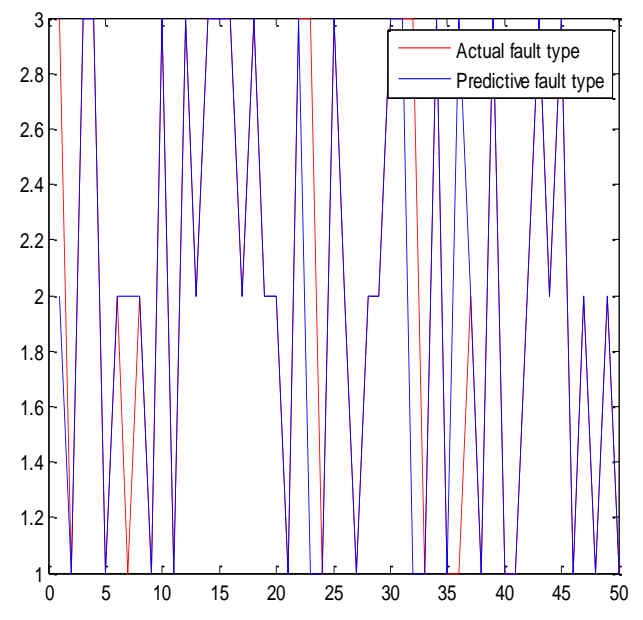

Fig. 6 Comparative results

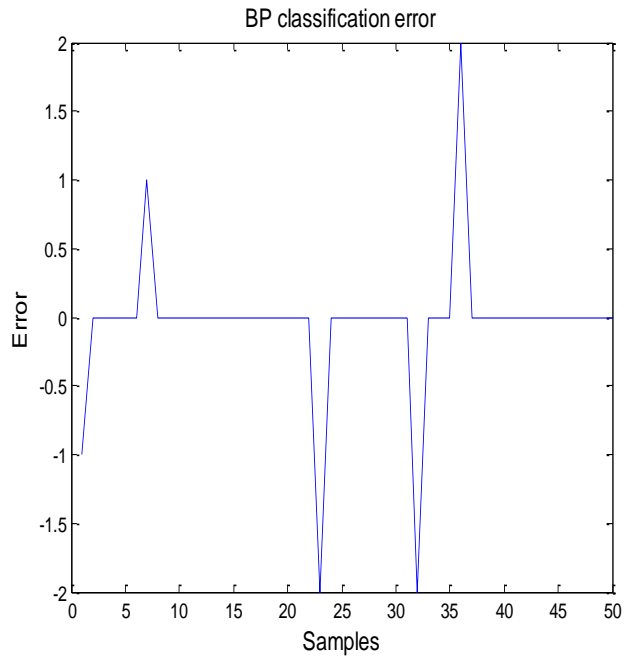

Fig.7 classification error

Analysis: the BP neural network classification accuracy rate:0.8043;0.8043;0.9256;

form the fig. 6 and fig. 7 we can see that the 50 test samples only 3 sample is inaccurate classification, so the network model application is effective.

(4)Get 6 groups bearing fault data for test, the results shown in Table 2: 
Table 2 diagnostic results

\begin{tabular}{|c|l|l|l|l|l|l|l|}
\hline samples & \multicolumn{3}{|c|}{ Test results } & \multicolumn{3}{|c|}{ Expected output } & result \\
\hline 1 & 0.9711 & 0.0002 & 0.0287 & 1 & 0 & 0 & normal \\
\hline 2 & 0.9810 & 0.0015 & 0.0175 & 1 & 0 & 0 & normal \\
\hline 3 & 0.0131 & 0.9797 & 0.0072 & 0 & 1 & 0 & inner fault \\
\hline 4 & 0.0181 & 0.9729 & 0.0090 & 0 & 1 & 0 & inner fault \\
\hline 5 & 0.0109 & 0.0092 & 0.9799 & 0 & 0 & 1 & outer fault \\
\hline 6 & 0.0089 & 0.0117 & 0.9794 & 0 & 0 & 1 & outer fault \\
\hline
\end{tabular}

Analysis: the table 2 shown that use of Wavelet Packet-BP neural network for fault diagnosis, the actual results and predicted fault diagnosis has consistent. It indicates that the method has a higher effectiveness.

\section{Conclusion}

Through the above experiments results it can be seen, using wavelet packet decomposition and BP neural network combined method for generator rolling bearing fault diagnosis.Achieve an accurate extraction of the wind turbine bearing normal state inner ring fault and outer ring fault feature vectors and effective classification; Wavelet packet suit for dealing with non-stationary signals, can accurately and effectively extract fault feature vectors; The extracted fault feature vectors as network input, training BP neural network, then the test samples are sent to the trained neural network, the accuracy of the classification rate reach to $93.3333 \%$, can achieve the accurate identification of the type of fault. Fault diagnosis based on wavelet packet decomposition and BP neural network, it can effectively improve fault diagnosis fast, effectiveness and accuracy.

\section{References}

[1] Wang Xianfeng, Li Hongtao, Barkhausen noise of Nondestructive Testing Technology, Bearing. 8 (2003) 27-28.

[2] Zhou Xiaoyong, Ye Yinzhong. Wavelet Analysis in Fault Diagnosis, Control Engineering, 13 (2006) 70-73.

[3] Xie Fangfang. Support Vector Machine for fault diagnosis[D].Hunan University, 2006.

[4] Yang Shuzi, Shi Tielin, Knowledge-based diagnostic reasoning, Tsinghua University Press, BeiJing, 1993.

[5] Mingshe W, Guoyong L, FNN-based intelligent fault diagnosis for ship diesel engine, Computer Simulation. 12 (2007) 159-162.

[6] Huang Jianhong, Jiang Qingnian and Zhang Xunhua, Wavelet Envelope Analysis Based on Wavelet Packet Energy Feature and Its Application in Failure Diagnosis of Rolling Bearing, Journal of Nanchang University: (Natural Science). 30 (2006) 402-405.

[7] Zhou Wei, Wavelet Analysis based on MATLAB, Xi'an University of Electronic Science and Technology Press, Xi'an, 2010. pp. 21-30.

[8] Xu Xianghe. Customer classification method based on BP ANN[D]. Nanjing: Nanjing University of Aeronautics and Astronautics, 2004.

[9] Zhou Zhihua, Cao Cungen, Neural Network and Its Application, Tsinghua University Press, Beijing, 2004. 